\title{
The Imminus Travel and Tourism Intranet
}

\author{
G. Edwards, C. Dawes, K. Kärcher
}

Paper presented at the 5th International Conference on Information and Communication Technologies in Tourism - ENTER, Istanbul, Turkey, 21 - 23 January 1998

\begin{abstract}
Viewdata (i.e. videotex) has been the underlying technology for electronic package holiday distribution in Britain for two decades. Increasingly, however, there is a need to move away from this technology platform towards a more modern and flexible electronic commerce environment in which travel and tourism companies can trade. The emergence of Internet-based and other technologies as well as changed customer demand make it more and more necessary for British travel and tourism companies to adapt to these changes and adopt the new technologies. Imminus Limited, one of the two main reservation system companies for the package holiday business in the UK, is proposing a migration from viewdata to a new technology platform, the Imminus Travel and Tourism Intranet. (It should be noted that this intranet is in fact both an intranet as well as an extranet.) This paper outlines, in case study form, key issues related to the development and implementation of this new industry trading platform, which is currently being piloted in co-operation with travel agents and tour operators.
\end{abstract}

\section{Introduction}

During the late 1970's and early 1980's, automated sale of package holidays was introduced in Europe on a larger scale with the implementation of national videotex-based reservation systems, such as START in Germany and Istel, Prestel and Imminus' Fastrak in Britain. The three British videotex systems are also referred to as viewdata.

Today, in the latter half of the 1990's, despite the emergence of new advanced technologies such as Internet-based technologies and multimedia (for example Kärcher, 1997a; McNeill, 1997), viewdata remains the main technology platform for the distribution of holiday packages in Britain.

Increasingly, however, travel and tourism principals, tour operators, travel agents and other travel and tourism organisations in Britain perceive the need to replace viewdata by new technologies. If these travel and tourism companies do not adopt the new technologies, certain customer demand will not be met and customers will increasingly be lost to those operators which embrace the new technologies (Datamonitor, 1997; Kärcher, 1997a).

Obviously, there is a reluctance to discard major investments made in viewdata technologies. In addition, there is little consensus in the industry on which new technologies to adopt. In this paper, a solution is presented for the migration of the British travel and tourism industry from viewdata to a new technology platform, referred to as a travel and tourism intranet. In essence, the solution proposed allows for a co-existence of viewdata and new, mainly Internet-based technologies, thereby enabling travel and tourism companies to continue using their present and proven viewdata systems, while also giving them the opportunity to cost-effectively adopt new technologies at the same time.

The Imminus Travel and Tourism Intranet combines both networking and business services and is designed to provide the benefits of the Internet, and its associated technologies, within an environment which can support the very specific business processes and practices of the travel and tourism industry. This intranet has been piloted during September and October 1997, with the first live booking having been made on 15 October 1997 between the travel agent Apollo Travel and the tour operator Crystal Holidays. A limited roll-out is taking place during late 1997 and early 1998, with a full roll-out planned for the following months. 
The term 'intranet' is used here to describe the implementation of a communications facility and business services utilising Internet technologies, but with access limited to authorised users. In the case of the Imminus Intranet, authorisation is limited to members of the travel and tourism trading community, as well as some organisations outside the core industry such as technology suppliers and insurance companies.

The 'intranet' discussed here is in fact both an 'intranet' as well as an 'extranet'. It is an intranet as it is owned and operated by a single organisation, i.e. Imminus Limited. It also is an extranet as the network links a wide community of travel and tourism organisations. However, given the less common use (and possibly knowledge) of the term 'extranet' within the travel and tourism industry, the term 'intranet' is used for marketing purposes and, for reasons of consistency, also throughout this publication.

Imminus' approach to the development of one of the key elements of the new electronic commerce platform - the reservation system front-end - is described in particular in this paper. The front-end is a value added service, which provides travel agents with a new generic interface (based on browser technologies) with which to make holiday bookings, and which gives tour operators and principals a relatively low cost distribution channel to the retail market.

\section{Main Characteristics of the Imminus Intranet}

Imminus Limited was founded by The Thomas Cook Group as Travinet Limited in the early 1980's, based on an internal information technology division. After a take-over of Thomas Cook by the Midland Bank PLC in 1983, Travinet was renamed Midland Network Services (MNS). The Midland Bank itself was acquired by the Hong Kong Shanghai Bank in the early 1990's. While Thomas Cook was sold to WestLB in June 1992, MNS was taken over in a management buyout in July 1993. In March 1994, the company was renamed Imminus (Latin, essentially meaning 'imminent'). General Cable PLC acquired Imminus in March 1997. General Cable is one of the largest companies in the United Kingdom according to market capitalisation and is listed on the London and New York stock exchanges. Since its acquisition, Imminus is being turned into the core of the newly formed General Telecom Limited, which covers all business-to-business interests of the General Cable group of companies.

In late 1997, Imminus controlled an estimated 50\% of the on-line holiday reservations market in Britain. Imminus owns and manages two extensive networks in the UK - an X.25 and a Frame Relay network -, and is in the process of implementing a high-speed ATM (asynchronous transfer mode) backbone network. In late 1996, a strategic business decision was taken by the senior management at Imminus to develop and implement the Imminus Travel and Tourism Intranet.

The world-wide Internet offers a number of benefits to the travel and tourism community, such as low cost, ease of use, multimedia presentation, hypermedia links, and world-wide diffusion (Kärcher, 1997b). However, the Internet has a number of key drawbacks when used particularly for business-tobusiness relationships. While the Internet is in principal open to every user, similar to a public transport system, intranets and extranets are only open to closed user groups. Resulting from this, the main benefits of intranets and extranets, when compared to the Internet, are:

- Security: Intranets and extranets offer a higher degree of security to their users.

- Guarantee of service, particularly speed: As intranets and extranets are managed services, they provide a guaranteed service and typically much higher speeds.

In addition, guarded through secure 'firewalls', intranet and extranet users can have access to the Internet and the services offered on it, and, thus, are able to also enjoy the benefits of the world-wide 
Internet. Due to these advantages, intranets and extranets are increasingly being implemented for business-to-business relationships in different industries (Moran, 1997).

In addition to the general benefits of intranets and extranets, the primary characteristics and advantages of the Imminus Travel and Tourism Intranet are:

- Exclusivity, specialisation and optimisation: The Imminus Intranet is exclusive to the travel and tourism community and meets the particular needs of the industry with products and services that are clearly differentiated from the generic services available from a multitude of telecommunications and IT suppliers. It also supports the high degree of seasonality and the (growing) need for seven days per week business activity. The intranet is optimised to allow accessibility, bookability and information provision tailored to the travel and tourism industry, thereby reducing valuable time spent by travel and tourism companies searching for this information.

- Service level: The Imminus Intranet is a managed service with 24-hour, 365-days per year support, management and maintenance through the Imminus Network Management Centre in Peterborough. This service includes high quality security, disaster recovery, hot restart, and back-up capabilities.

- Co-existence of established and new technologies: The single most important and unique aspect of the Imminus Intranet is that it supports the co-existence of viewdata and new technologies, and the migration from viewdata to a more sophisticated and effective electronic commerce platform for the distribution of travel and tourism products.

\section{Front-End Business Description}

The front-end is effectively a value added service to enable the distribution of leisure travel and tourism products between principals, tour operators and travel agents. Its key significance to the travel and tourism industry is that it enables a (relatively easy and low cost) migration from the viewdata infrastructure, which currently enables the trading process in the United Kingdom, to the more costeffective and advanced technologies which comprise the intranet.

The front-end enables the travel and tourism trading community to maintain the 'many-to-many' trading relationships (Fig. 1) currently supported by the use of viewdata through two key mechanisms:

- Travel agents get a generic (but customisable) user interface which allows the entry of booking details; just as viewdata currently permits, but with enhanced navigation and information facilities.

- A protocol conversion module translates information entered at the user interface into the appropriate EDI message formats for transfer to host systems to initiate availability enquiries or reservations. The results of those transactions are then translated by the conversion module for display to the user. This module allows connectivity with the widest possible base of tour operators' and principals' existing reservation systems.

More specifically, the module allows for co-existence of viewdata and IP (Internet Protocol) based technologies. 


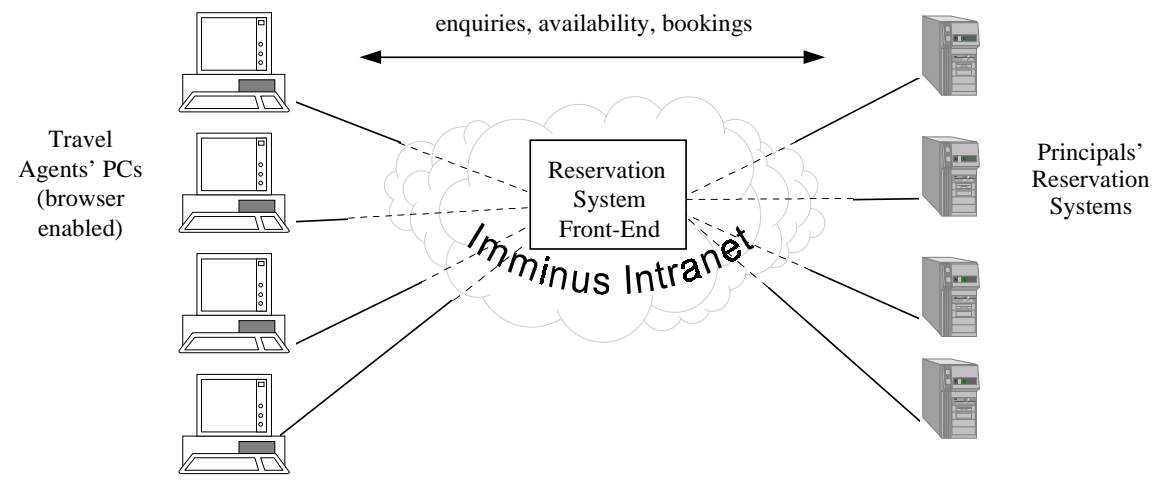

Fig. 1. Travel and Tourism Industry Business Process Support

The authors anticipate that the migration from viewdata to intranet bookings will take, from the completed system pilot, between 18 months and two years for most travel agents, and even longer for tour operators and principals. Imminus has, therefore, incorporated various features into the front-end system, user interface, and required hardware configurations to ensure that the cross-over period, when viewdata and the intranet co-exist, can be managed with the minimum of problems to travel agents, tour operators and principals.

Some of the front-end's features include:

- Allowing the user interface to make viewdata bookings by embedding a videotex session within the browser.

- Defining low cost hardware and connectivity options, which give all travel and tourism companies the opportunity to access the front-end.

Specific benefits accruing to travel agents from using the front-end include the following:

- The ability to make multiple, simultaneous enquiries for a holiday using only one set of data entry screens.

- The ability to effectively link front and back-office systems and to gain improvements in managing customers and providing customer service.

- The front-end can take full advantage of the intranet's high network speeds to speed-up the booking process for agents.

- The front-end is configurable to match the business practice and commercial needs of agents. For instance, it allows automatic search on preferred supplier systems.

- The front-end is able to support further booking process features such as the inclusion of images and video-clips, but only if required. These features are particularly useful for more complex and, usually, higher value sales.

Specific benefits accruing to travel and tourism principals and tour operators from travel agents using the front-end include the following:

- Without the front-end, these organisations would have to set up bilateral communications and trading facilities with travel agents.

- The front-end leads to a significant reduction in distribution costs because of the low costs of the technology components (e.g. PC, modem, ISDN, TCP-IP protocols, browser, reduced PSTN costs) and their improved manageability.

- The front-end enables transaction charging to take place, providing principals and tour operators with a known cost of sale.

- The front-end generates detailed and accurate management statistics regarding booking transactions. 
- Principals and tour operators are able to give better support to the retailers in the form of customer specific sales and marketing information as well as customer service because of the improved user interface platform.

- The front-end provides a technological migration path suitable for the entire retail community, allowing principals and tour operators to maintain their market penetration whilst, in addition, enjoying the benefits of that technological change.

\section{Investment Rationale for Front-End Development}

- Supporting the intranet: The front-end is the linchpin service of the intranet as it supports, and therefore allows Imminus to take revenue from, the primary transactions of the travel and tourism industry, i.e. enquiries and bookings. Without the front-end, the intranet would not be a viable proposition.

- Competitive differentiation: The development of the front-end value added service reduces Imminus' exposure to the risk of commoditisation of 'vanilla' network provision.

- Competitive advantage: Imminus' main competitors have adopted an Internet-centric approach to the market which puts them at a disadvantage vis-à-vis the key features of the intranet (Section 2). The implementation of the front-end within the intranet is likely to put Imminus in a good position to capture a high market share of booking transactions.

\section{Front-End Development Requirements}

The front-end has to manage a very large volume of transactions between travel agents, tour operators and principals. In order to perform this task and to support the commercial requirements of Imminus, a sophisticated software application is required. The functionality of the application is divided among four main software modules (Fig. 2).

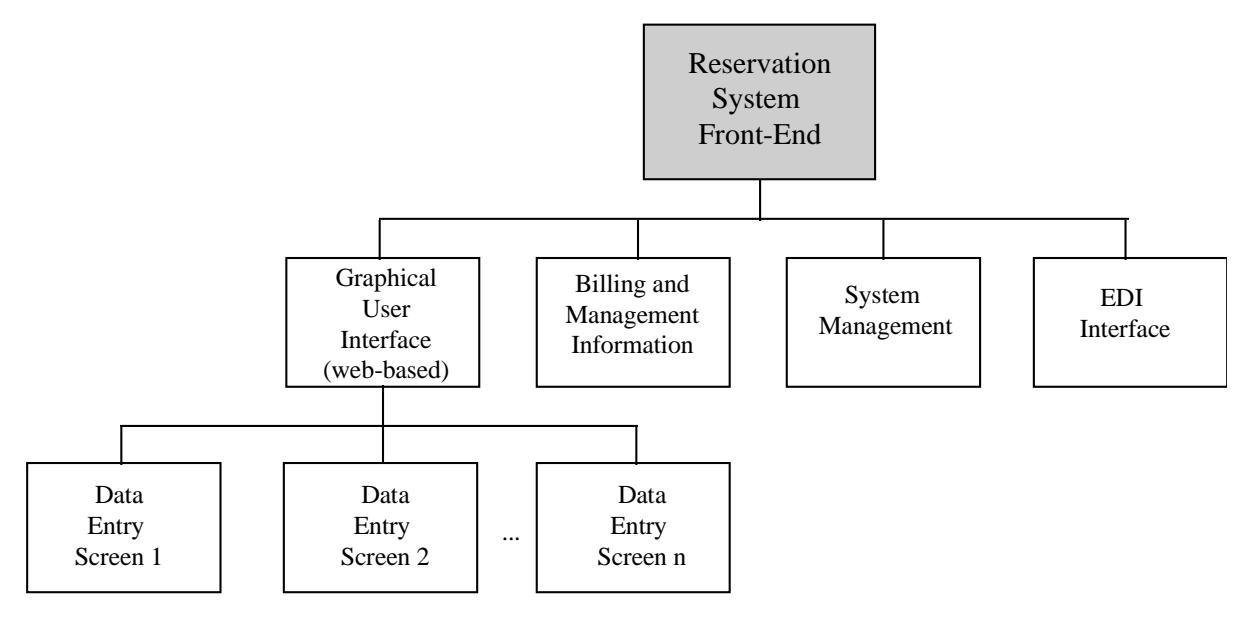

Fig. 2. Reservation System Front-End Design

The front-end development process includes a detailed design specification. Development has been 'back-to-back' with the project to develop an Imminus Travel and Tourism Intranet demonstration system. The broad functionality of each of the front-end modules is outlined in the following. 


\subsection{Graphical User Interface (GUI)}

Browsers are loaded on travel agent PCs and workstations to allow access to the front-end service. The browsers are pre-configured to ensure both that users start off at the appropriate point in the intranet, and that a level of default functionality is provided. The interface consists of a number of data entry screens (effectively web pages), which allow the entry of the appropriate details to book holidays or access holiday services. Input required includes passenger, departure and destination details. Multiple, simultaneous enquiries can be made using a single, completed set of entry screens. The GUI is configurable to allow travel agents to, for instance, enquire on a list of preferred hosts, or select specific operators for enquiries. It also is capable of managing at least 10 response windows to display availability details returned from the tour operator systems. High quality graphics, images, video clips and audio clips are also catered for in the GUI. It is important to note, however, that these features are travel agent configurable to ensure that they are only used where they are beneficial. For instance, it may not be appropriate to show on-line images of resorts or hotels to support the sale of a 'bucket-and-spade' holiday as this would slow the sales process. However, for higher margin products, such as cruises or safaris, the provision of extra information and pictures may mean the difference between a sale and a no-sale, or may lead to the sale of 'travel-extras' at premium prices.

\subsection{Billing and Management Information}

Imminus will charge hosts per completed transaction (by booking or by PAX). It is absolutely vital, therefore, that transactions can be trapped and passed to the billing module. The billing module can then interface into company financial systems. Audit trails of transactions are also required to allow accurate reporting and management information provision on system activity for both Imminus and its customers.

\subsection{System Management}

System management requirements for the front-end service have both a travel agent and an internal dimension. From a travel agent's point of view, the front-end service (and the access infrastructure needed to support it) is relatively complex. To reduce this complexity, Imminus will remotely manage travel agents' workstations, browsers and network access equipment. Internally, Imminus will be able to dynamically re-configure the core front-end service either for specific customers or as a whole.

\subsection{EDI Interface}

The EDI (electronic data interchange) module is the interface between the web-browser based GUI and the host reservation systems. Structured messages generated by the hosts are received by this module and interpreted for display. Data entered into the GUI goes through the interface for translation into a structured message format. This module is capable, over time, of incorporating the message formats of the majority of the reservation systems used by principals and tour operators. The structured message is based on the industry-wide agreed RESCON (short for 'reservation confirmed') EDI format (Jolley, 1997). 


\section{Front-End Operational Requirements}

The front-end is supported by a complex networking and computing infrastructure (Fig. 3), which is designed to provide resilience and security to users, as well as flexibility to cope with fluctuations in usage on both a daily and a seasonal basis. The intranet provides interconnectivity between Imminus' X.25 packet switched network, its Frame Relay network, its ATM backbone network, the publicswitched telephone network (PSTN) and ISDN, as well as the Internet. The intranet is protected by a firewall to prevent unauthorised access from the Internet. Intranet users have access out to the Internet, but lose security and performance guarantees in so doing.

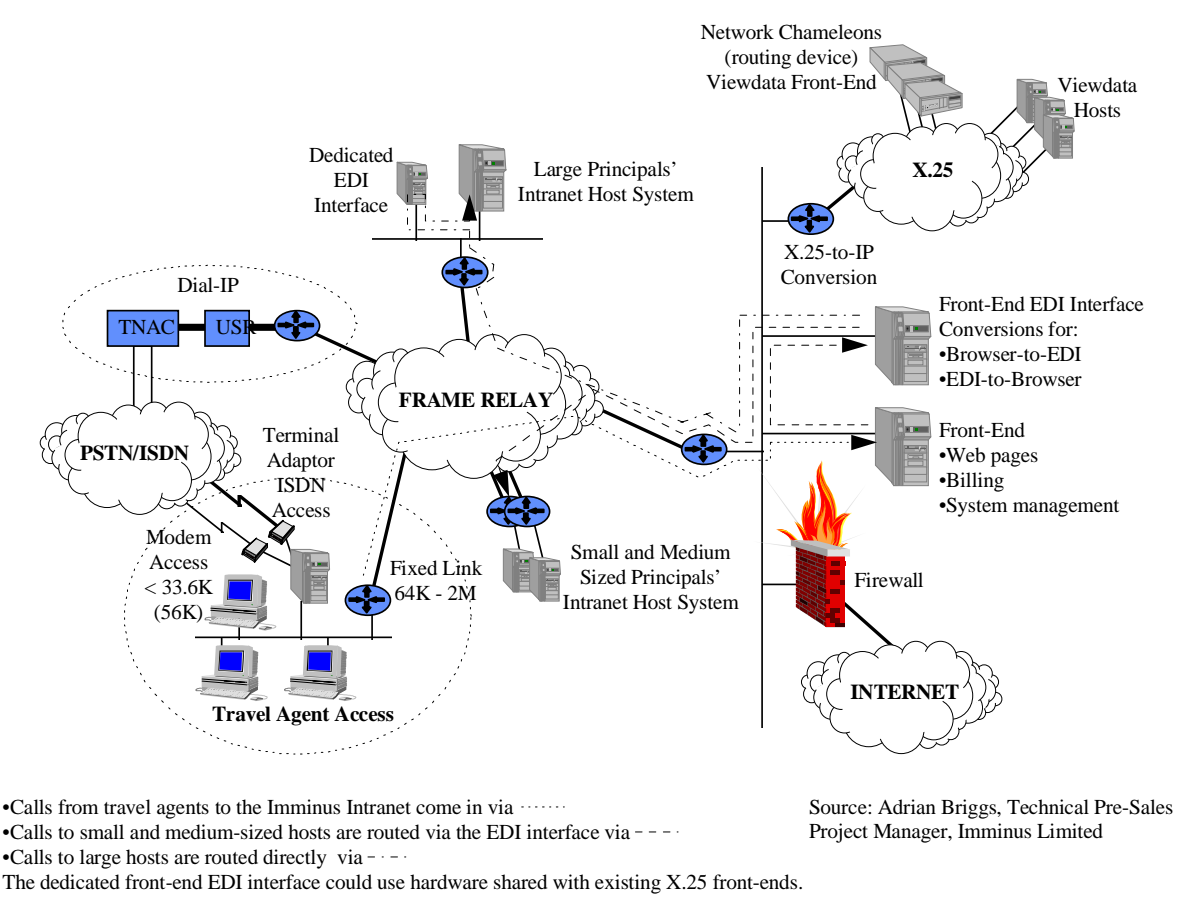

Fig. 3. Imminus Intranet and Reservation System Front-End

Principal and tour operator host system connectivity to the intranet is supported by the use of router devices. Large principals and tour operators need to have a dedicated EDI interface to support the translation of structured EDI messages into host system message formats. Small and medium-sized principals and tour operators have the option of sharing an EDI interface.

Travel agents access the service from intranet-enabled PCs and workstations. Three access options have been defined:

- MRS (Managed Router Service) access, supporting multiple users.

- ISDN (Integrated Services Digital Network) access, supporting multiple users.

- Dial-up IP (Internet Protocol) access, achieved by up to four PC users sharing a high speed modem. 


\section{Conclusions and Outlook}

There is little doubt that the travel and tourism industry in the United Kingdom has to adopt new technologies for holiday distribution. The question is when and how this will happen. In this paper, the authors discuss Imminus' approach to introducing new technologies which offer the industry a clear route forward and which minimise the risks, problems and costs associated with change. The most potent obstacle to change may, however, lay in the possible inability of members of the industry to adopt a more co-operative approach with each other to move to a new technology platform.

From Imminus' perspective, the success of the front-end would support the development and marketing of a portfolio of intranet services illustrated in Fig. 4. These services are aimed largely at niche markets within the travel and tourism industry. Their relationship with the front-end service is somewhat symbiotic, and their independent viability cannot be guaranteed without the existence of the front-end. However, they also enhance the attractiveness of the front-end service particularly during the migration period between viewdata and intranet bookings.

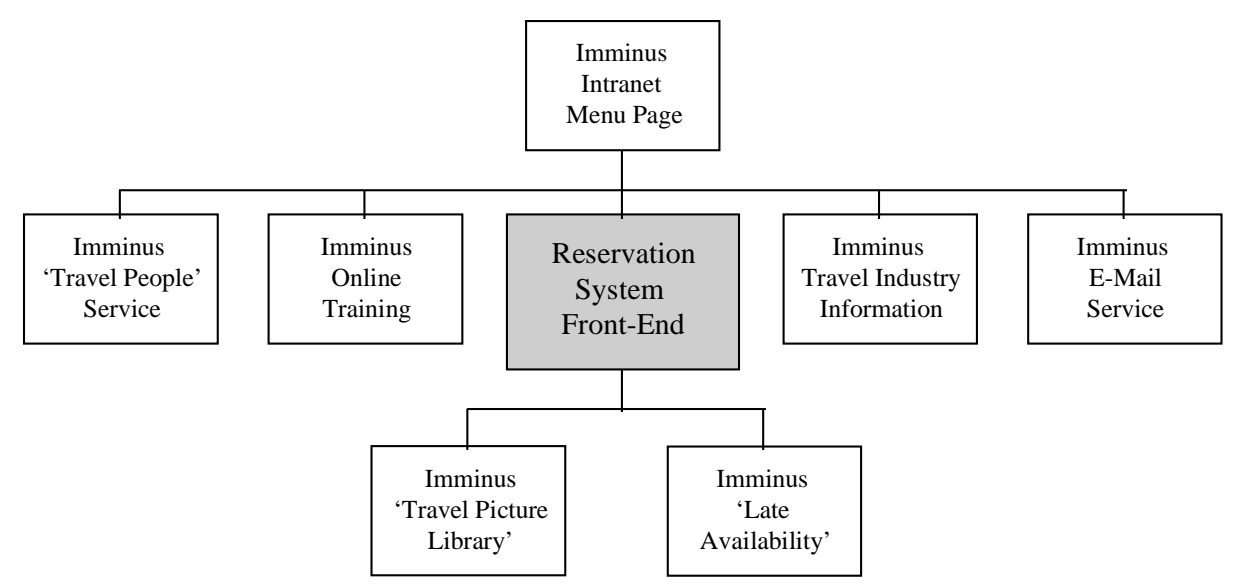

Fig. 4. Proposed Intranet Value Added Services

The proposed intranet services can be grouped into two broad categories:

- Services which enhance the functionality of the front-end, and thereby the booking or sales process, by providing additional transactions or information at the point of sale. Examples:

- A 'Travel Picture Library' service - which stores images of resorts, hotels, tourist attractions etc. in a central database for use in brochures and other publications, and for display on travel agents' screens. This facility removes the need for hosts to overhaul their systems to cope with images.

- A generic late availability database - which provides travel agents with a single source of late availability holidays to sell, whilst providing hosts with a mechanism to off-load unsold stock in a manner and in an environment which does not undermine their market positioning.

- Services which support a range of other processes within the travel and tourism industry. Examples:

- A recruitment and career counselling service called 'Travel People' - which is aimed at individual workers within the travel and tourism industry. The intention is to link training records to the production of $\mathrm{CVs}$ and to provide personalised, but database driven assistance to individuals in planning and developing their careers.

- An on-line interactive training service - which Imminus has established in partnership with The Travel Training Company (formerly the ABTA National Training Board). On-line 
training should provide better access to training courses and reduce the cost of training. The interactive nature of the courses also has the potential of improving the efficacy of training by making it more interesting and, therefore, increasing knowledge retention.

- Travel and tourism industry specific news and information services - designed to support various segments of the industry.

- An e-mail service - which provides an interface between the intranet and viewdata systems to support cross industry communications and which includes a comprehensive directory of industry participants.

\section{References}

Datamonitor (1997). New Technologies in Travel \& Tourism: Evolving distribution channels. Datamonitor, London, England.

Jolley, R. (1997). 'Fans of Rescon Look for Operator Take-Up: The Travel Technology Initiative believes the trade is gradually waking up to the advantages of using Rescon - electronic data interchange - to speed the booking process and cut costs.' Travel Trade Gazette UK \& Ireland, 12 March, p. 26.

Kärcher, K. (1997a). Reinventing the Package Holiday Business: New information and communication technologies. Deutscher Universitäts-Verlag (DUV), Wiesbaden, Germany.

Kärcher, K. (1997b). 'Sonne, Sand und Spass? Bitte hier anklicken!' Neue Zürcher Zeitung (NZZ), 137, 17 June, p. B10.

McNeill, L. (1997). Travel in the Digital Age. Bowerdean Publishing Company Limited, London, England.

Moran, N. (1997). 'Business-to-Business Links: Now the attention turns to extranets.' Financial Times, 4 June, pp. V - VI.

\section{About the Authors}

Gareth Edwards is Business Development Manager, Chris Dawes is Commercial Director, and Dr. Karsten Kärcher is Account Director; all at Imminus Limited, Peterborough, England (contact: Karsten.Karcher@ GenesysInformation.com). 